\title{
E-maturity (e-M): construção e elaboração do questionário de autoavaliação da Gestão Tecnopedagógica
}

\author{
Herik Zednik Rodrigues ${ }^{1}$, Liane M. R. Tarouco ${ }^{1}$, Luis R. Klering ${ }^{2}$ \\ ${ }^{1}$ Programa de Pós-graduação em Informática na Educação - Universidade Federal \\ do Rio Grande do Sul (UFRGS) \\ ${ }^{2}$ Faculdade de Administração -Universidade Federal do Rio Grande do Sul \\ (UFRGS) \\ herik.zednik@ufrgs.br, liane@penta.ufrgs.br, rsf2708@via-rs.net
}

\begin{abstract}
.
The aim of this study is to describe the proposed development of the assessment tool and monitoring of management-pedagogical. The model systematizes an instrumental analysis of the level of e-maturity, in relation to the strategic and effective use of ICT to improve educational outcomes. In this sense, the instrumental collaborates in identifying the uses and appropriations of ICTs in schools, from the perspective of pedagogical practice. The instrument was based on tools for ICT Self Review Framework, originally developed in England by Naace Advancing Education Through ICT and the CM360 model proposed by Franco and Santos (2009), which is a framework for analyzing and evaluating management maturity content of an organization.
\end{abstract}

Keywords: e-maturity. Management. Technopedagogical

\begin{abstract}
Resumo.
O objetivo desse estudo é descrever a proposta de desenvolvimento da ferramenta de avaliação e acompanhamento da gestão tecnopedagógica (e-Maturity). O modelo sistematiza um instrumental de análise do nível de maturidade, em relação ao uso estratégico e eficaz das TIC na melhoria dos resultados educacionais. Nesse sentido, o instrumental colabora na identificação dos usos e apropriações das TIC nas escolas, numa perspectiva da prática pedagógica. O instrumental foi elaborado com base nas ferramentas E-learning Maturity Model - eMM (Marshall e Mitchell, 2004), Self Review Framework for ICT, originalmente desenvolvida na Inglaterra pela Naace - Advancing Education Through ICT e no modelo CM360 proposto por Franco et al (2009), que é um framework de análise e avaliação de maturidade da gestão de conteúdo de uma organização.
\end{abstract}

Palavras-chaves: maturidade eletrônica. Gestão. Tecnopedagógica.

\section{Introdução}

O panorama atual reflete um mundo com grande intensidade no fluxo de informações, em constante mutação nas mais diversas áreas, "onde o conhecimento é um recurso flexível, fluido, sempre em expansão e em mudança" (Hargreaves, 2003, p. 33 apud Coutinho e Lisboa, 2011). Acompanhar essa evolução não tem sido tarefa fácil para a escola, que por sua vez busca se encontrar nesse mundo desterritorializado,

[...] onde não existem barreiras de tempo e de espaço para que as pessoas se comuniquem. Uma nova era que oferece múltiplas possibilidades de aprender, em que o espaço físico da escola, tão proeminente em outras

V. $11 \mathrm{~N}^{\mathrm{o}}$ 1, julho, 2013 
décadas, neste novo paradigma, deixa de ser o local exclusivo para a construção do conhecimento e preparação do cidadão para a vida ativa (Coutinho e Lisboa, 2011).

O contexto atual implica em urgente ressignificação do papel da escola e de seus atores. A subutilização (tanto no aspecto administrativo quanto pedagógico) do significativo volume de recursos tecnológicos presentes na escola denuncia 0 descompasso entre escola/sociedade/tecnologia. Nesse sentido, “[...] o conjunto de habilidades exigidas dos gestores escolares está cada vez maior e abrange várias áreas do conhecimento. Essas novas habilidades trazem à tona a necessidade de uma maturidade eletrônica" (Zednik et al, 2012) aplicada ao contexto escolar, como forma de atender a essa nova demanda e caminhe para ações que evoluam em direção ao uso eficiente e estratégico das TIC. O desafio é incorporar os novos recursos em atividades da rotina escolar de forma pedagógica, ou seja, gerenciar de forma criativa e inovadora a tecnologia para que esta colabore na melhoria dos resultados educacionais.

Nesse mundo transitório, “[...] urge criar uma „ponte" que permita conectar as organizações à realidade complexa em que se encontram, permitindo uma análise abrangente dos fatores que interessam ao seu público alvo. Surge daí o conceito de maturidade e estratégia de ação." (Franco et al, 2009, p. 6).

Nesse sentido, cabe às escolas entenderem seu posicionamento em relação à gestão tecnopedagógica ${ }^{\mathrm{i}}$, articulando ações coletivas que caminhem para um gradativo e holístico amadurecimento.

No contexto deste trabalho, e-maturity se traduz na evolução da "capacidade e potencialidade da organização escolar de tomar decisões estratégicas e utilizar de forma eficaz a tecnologia para melhorar os resultados educacionais (Zednik et al, 2012, p.7)".

Com base na realidade local (Escolas públicas do Estado do Ceará), no Elearning Maturity Model - eMM (Marshall e Mitchell, 2004), nas ferramentas Self Review Framework for ICT, originalmente desenvolvida na Inglaterra pela Naace Advancing Education Through ICT e no modelo CM360 proposto por Franco, Santos e Terra (2009), que é um framework de análise e avaliação de maturidade da gestão de conteúdo de uma organização, foi adaptado, estruturado, aperfeiçoado e consolidado o questionário de autoavaliação da Gestão da $\boldsymbol{e}$-Maturity (e-M), que favorece o acompanhamento e avaliação da maturidade tecnopedagógica de escolas públicas.

Segundo Yanaze (2009, p. 74) tecnopedagogia se traduz no "sentido de considerar que o ser tecnológico se forma através da tecnologia e que esta é inerente ao contexto do ser. Ou seja, as tecnologias, ou melhor, as tecnopedagogias ampliam o processo de formação do homem que é essencialmente tecnológico". Desta forma, se caracteriza como um processo que compreende a pedagogia em confluência com a tecnologia na formação do ser tecnológico.

\section{2. e-M: aporte teórico}

Este trabalho fornece uma descrição dos processos e das práticas que sustentam o questionário de autoavaliação da Gestão da $\boldsymbol{e}$-Maturity (e-M), proposto para a pesquisa de doutorado intitulada $E$-maturity: gestão da tecnologia em função da melhoria do desempenho educacional, desenvolvida no Programa de Pós-Graduação em Informática na Educação (PPGIE), da Universidade Federal do Rio Grande do Sul. A construção do questionário teve como referência os questionários acima citados por serem concernentes à mesma temática.

Para fundamentar a ideia evolucionária de gestão buscamos suporte na Teoria da Maturidade-imaturidade, de Chris Argyris (1969); no Modelo de Maturidade de 
Capacidade de Software - CMM, proveniente da Engenharia de Software; e nas Perspectivas de Transformação de Paulo Roberto Motta (1999).

Segundo Argyris (1968), o indivíduo imaturo apresenta as seguintes características: ser passivo, dependente, postura de subordinado, entre outras. Ao evoluir para maturidade, apresenta "características de um ser ativo, independente, com postura mais flexível, interesses mais profundos e variados, comportamento mais complexo e perspectiva mais longa, maior consciência e controle" (Argyris, 1968 apud Zednik, 2012, p. 4). Esta concepção fortalece e encoraja mudanças organizacionais, pois "está implícita a ideia do homem valorizado e identificado com os objetivos da organização, possibilitando, desta maneira, alto grau de satisfação no trabalho e maior produtividade" (Pérez-Ramos, 1980, p. 61 apud Martinez e Paraguay, 2003).

O Modelo de Maturidade de Capacidade de Software - CMM defende que a maturidade de uma organização está relacionada à sistematização das práticas necessárias para sua efetivação, isto se traduz através “de políticas, padrões, cultura corporativa, práticas gerenciais, estruturas organizacionais" (Bruno, 2008 apud Zednik et al, 2012, p. 6).

Motta (1999), em seu livro Transformação Organizacional, enfoca como funciona e quais características tem uma gestão organizacional mais evoluídadesenvolvida. Segundo o autor, as mudanças organizacionais tendem a ocorrer em seis importantes perspectivas: contextual, estrutural, tecnológica, humana, cultural e política. Dentre as características de organizações mais desenvolvidas, destaca: gestão por processos ou projetos; uso da tecnologia de maneira interdisciplinar e integrada; organização por equipes multifuncionais; busca de resultados por caminhos não únicos; valorização da autonomia relativa e do autocontrole; aderência à missão organizacional; valorização da criatividade, do talento, das habilidades múltiplas e da personalidade de cada um; valorização da participação, das decisões por colegiado e do poder distribuído, dentre outras características distintivas.

\subsection{E-learning Maturity Model (eMM)}

A versão 2.3 é a mais recente do E-learning Maturity Model (eMM) ${ }^{\mathrm{ii}}$. O processo eMM está dividido em cinco áreas: Aprender, Desenvolvimento, Apoio, Avaliação e Organização. O quadro utilizado nesta análise é baseado no Capability Maturity Model. A ideia subjacente é a de que a capacidade de uma instituição para ser eficaz em uma determinada área de trabalho é dependente da sua capacidade de participar em processos de alta qualidade que se consolidam com ações maduras. As características de uma instituição que permite processos de alta qualidade são, até certo ponto, capazes de serem separadas a partir dos detalhes do trabalho real empreendido e varia dependendo das circunstâncias. Esta separação significa que a análise pode ser feita de forma independente das tecnologias selecionadas e pedagogias aplicadas, permitindo assim uma comparação significativa em todo o setor (Marshall, 2006, p.5).

Nesse contexto, (Ibid, 2006) Capability, refere-se à capacidade de uma instituição para garantir que a concepção de desenvolvimento e implantação da $e$ learning atenda às necessidades dos diversos segmentos presentes na escola (alunos, professores, funcionários, pais e instituição). Capability inclui a capacidade de uma instituição para manter o apoio da e-learning no ensino como demanda crescente e mudança pessoal.

Os processos utilizados no e-MM foram desenvolvidos a partir de "Sete Princípios" de Chickering e Gamson (1987) e "Qualidade na Linha benchmarks" (IHEP 2000). O conceito de níveis foi baseado na avaliação da primeira versão do e-MM (Marshall e Mitchell, 2006). O uso de níveis implica um modelo hierárquico onde a 
capacidade é avaliada e construída na forma de camadas. A ideia-chave subjacente ao conceito de níveis é a capacidade holística. Ao invés do modelo de mensuração de níveis progressivos, descreve a capacidade de um processo a partir de perspectiva sinérgica.

\subsection{Self Review Framework for ICT (Naace - Advancing Education Through ICT)}

Esta ferramenta online oferece um percurso estruturado para rever e melhorar o uso da Tecnologia nas escolas do Reino Unido. A autoavaliação Framework (SRF) ${ }^{\text {iii }}$ fornece uma estrutura para analisar o uso das TIC e seu impacto na melhoria da escola. O modelo é projetado para ajudar a rever o uso da tecnologia de forma estruturada, o que por sua vez irá ajudar a informar a escola sobre a estratégia de melhoria e planos.

O quadro é projetado para suportar todas as escolas e todos os aspectos são igualmente relevantes para escolas infantis, escolas especiais e escolas primárias ou secundárias. O quadro é revisto sistematicamente, após consulta com professores, diretores, e todas as principais partes interessadas. Uma comissão nacional constituída supervisiona a revisão e manutenção do quadro para garantir que ele permaneça atualizado e relevante, com mudanças nos padrões de qualidade, práticas e das TIC (Naace, 2006).

O SRF é composto por 6 (seis) elementos para análise: Leadership and Management; Planning; Learning; Assessment; Professional development e Resources. O modelo e-M, proposto nesse trabalho, baseou-se nessa divisão, porém, além de acrescentar um sétimo elemento, Gestão da E-segurança, realizou adequações, para ajustar o modelo à realidade local e à literatura sobre gestão organizacional, especialmente os modelos de Argyris (1969) e Motta (1999).

\subsection{Modelo CM360}

Apesar do modelo CM360 estar voltado, a princípio, para a análise e avaliação de conteúdo, o seu escopo pode ser pensado em diferentes contextos. Além de ser um modelo abrangente e objetivo, se diferencia por ser "uma ferramenta de análise do modelo organizacional de gestão do conteúdo, mas também um instrumento de planejamento e apoio para o desenvolvimento das competências e processos necessários a uma gestão eficaz" (Franco et al, 2009, p. 116).

O modelo propõe uma escala de avaliação da maturidade da empresa, direcionada por amplos critérios que permitem a organização e articulação harmônica das ações. Nessa proposta, a avaliação é o início do ciclo de monitoramento e evolução da gestão em uma organização. A aplicação do modelo se divide em 4 (quatro) fases: preparação, avaliação, análise e ação, conforme diagrama abaixo.

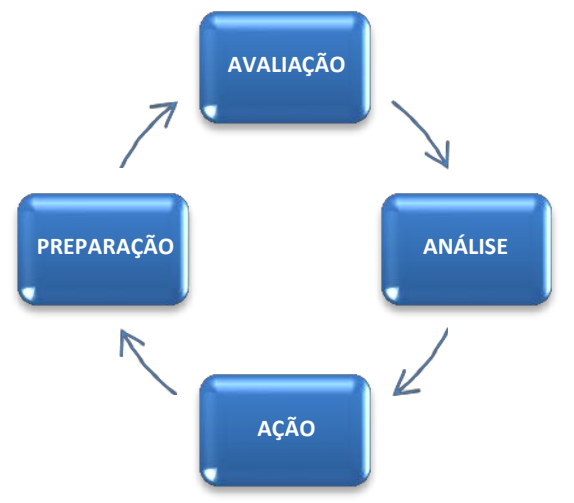

Figura 01 - Ciclo CM360

Fonte: Franco e Santos (2009), p. 137.

V. $11 \mathrm{~N}^{\mathrm{o}}$ 1, julho, 2013 
No contexto educacional, o resultado da aplicação deste modelo é que a avaliação final da iniciativa pode ser estudada e analisada pelos diferentes segmentos da escola, permitindo a elaboração de planos de ação coletivos e colaborativos, "ao mesmo tempo em que o gestor da iniciativa tem a visão em $360^{\circ}$ do projeto" (Ibid, 2009, p.124). Nesse sentido, o processo de avaliação deve ser contínuo, um novo processo se inicia ao final de cada ciclo de avaliação e aprimoramento, com o "intuito de avaliar a relevância das ações executadas e estabelecer um comparativo evolutivo entre os ambientes antes e depois do ciclo realizado" (Ibid 2009, p. 137).

\section{E-maturity (e-M): ferramenta de avaliação e acompanhamento da Gestão Tecnopedagógica}

O E-maturity (e-M) é um modelo de autoavaliação criada não apenas com o objetivo de oferecer dados que representem a maturidade atual da gestão da tecnologia no contexto escolar, mas principalmente para apoiar e orientar o gestor no planejamento de ações necessárias de aprimoramento do uso pedagógico das TIC, bem como a ordem de precedência adequada destas ações.

Via modelo proposto, o diretor ou comunidade escolar pode acompanhar possíveis mudanças na dinâmica escolar da Gestão Tecnopedagógica, procedente do monitoramento do nível de e-maturity. "Logo, o processo de avaliação se desdobra nas reflexões que levam à definição dos próximos passos de forma contundente e subsidiada pelos resultados e análise da avaliação" (Ibid, 2009, p. 137).

O questionário autoavaliativo construído para a pesquisa possui 4 (quatro) seções distintas:

a. Seção A - Corresponde aos dados da escola e perfil do Gestor;

b. Seção B - Corresponde aos dados do Grupo Focal;

c. Seção C - Corresponde ao nível de e-maturity (quadro de autoavaliação);

d. Seção D - Corresponde ao Footprint (Pegada da Tecnologia Educacional - TE).

A tela inicial do sistema identifica o usuário que acede ao recurso. A $2^{\mathrm{a}}$. tela corresponde ao cadastro onde o respondente preencherá algumas informações sobre escola pesquisada e sobre o respondente, com o objetivo de criar um login e senha para acessos posteriores, bem como, traz o termo de consentimento para utilização dos dados fornecidos na pesquisa, conforme apresenta a Figura 03.

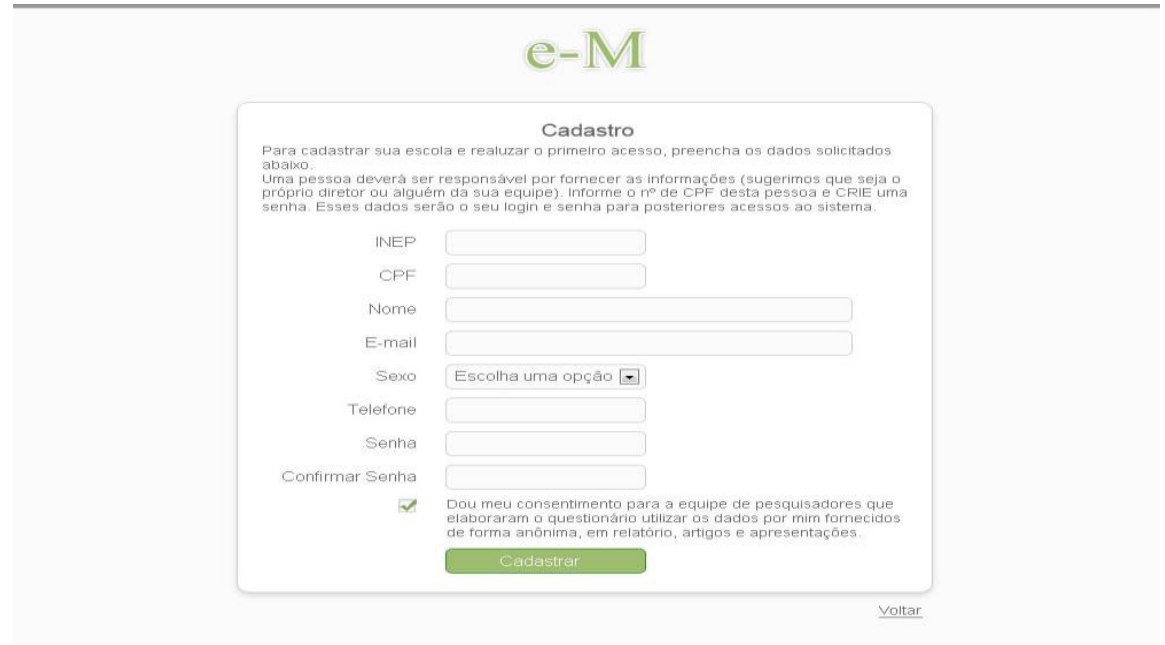

Figura 02 - Tela 02

Fonte: Zednik (2013) 
As telas 03, 04, 05, 06 e 07 correspondem à Seção A, onde se objetiva conhecer dados gerais da escola e o perfil do Gestor. Esta Seção subdivide-se em: Identificação; Ensino; Dados complementares; Diretoria e Indicadores.

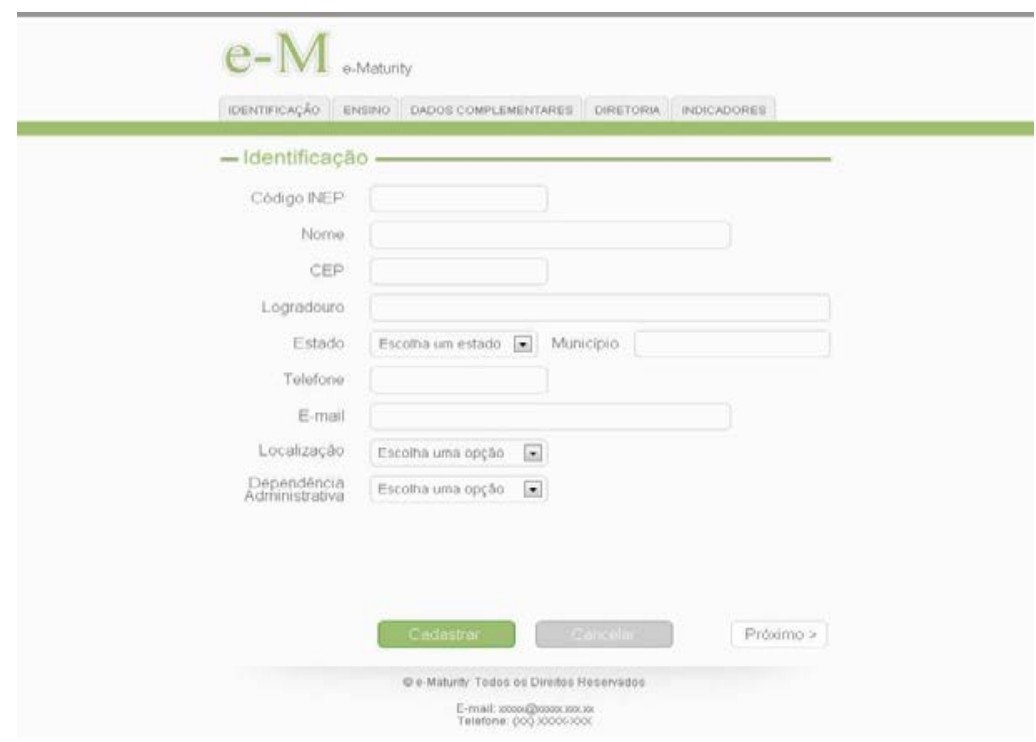

Figura 03 - Tela 03 - Identificação da Escola

Fonte: Zednik (2013)

A tela 08 corresponde à Seção $\mathrm{B}$, em que são inseridos os dados (nome, função e e-mail) do Grupo Focal participante da pesquisa. A Seção C corresponde ao nível de $e$ maturity, em que o respondente tem acesso ao quadro de autoavaliação da Gestão Tecnopedagógica, respondendo a um conjunto de questões que variam em uma escala do tipo Guttman, que vai de 1 a 5 .

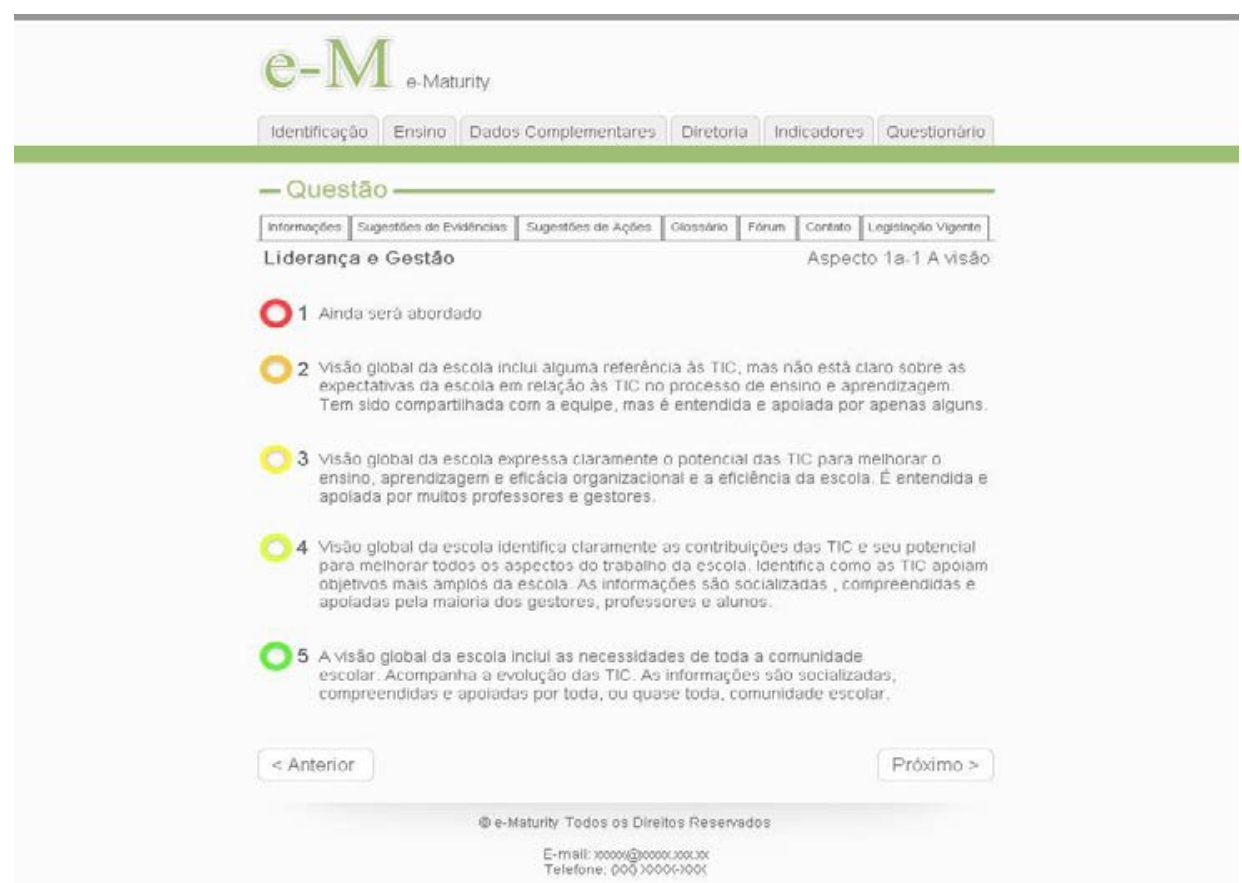

Figura 04 - Tela 09

Fonte: Zednik (2013) 
"Uma escala do tipo Guttman ou Cumulativa é composta por um conjunto de frases (itens) organizadas de forma hierárquica e em relação aos quais se pede ao sujeito avaliado que manifeste a sua concordância ou não" (Cunha, 2007, p. 26). A questão que alicerça a elaboração de uma escala tipo Guttman é a ordenação dos itens que garanta um crescendum na atitude, desde a menos abrangente até a mais ampla.

A escolha da escala de Guttman se justifica por esse modelo trabalhar melhor com questões hierárquicas e estruturadas, como os estágios evolucionários. Segundo Cunha (2007, p. 27), a medida da atitude é dada pelo padrão de resposta, isto é, a combinação de frases com que o sujeito que é avaliado concorda. Como as frases são organizadas de forma crescente, a formulação das frases, bem como a ordem pela qual são apresentadas, leva à ideia de acúmulo, de evolução de um estágio para outro.

Além das questões, a tela dispõe, para apoio ao usuário, dos seguintes elementos: informações acerca do aspecto analisado; sugestões de evidências; sugestões de planejamento de ações para melhoria do nível de maturidade; glossário, fórum, contato e a legislação vigente.

Neste trabalho, a escala é representada também por cores que correspondem à intensidade em que a situação descrita ocorre de acordo com o nível de maturidade avaliado pelo usuário, representando pontuações relacionadas à e-maturity com base em um modelo de semáforo, e apresenta a seguinte graduação: vermelho (nível 1), laranja (nível 2), amarelo (nível 3), verde claro (nível 4) e verde escuro (nível 5).

\begin{tabular}{|c|c|c|c|c|}
\hline $\mathbf{1}$ & $\mathbf{2}$ & $\mathbf{3}$ & $\mathbf{4}$ & $\mathbf{5}$ \\
\hline $\begin{array}{c}\text { Não respondeu/ } \\
\text { não sabe/ não } \\
\text { iniciou }\end{array}$ & $\begin{array}{c}\text { Fraco/ ainda não } \\
\text { começou/ fases } \\
\text { iniciais }\end{array}$ & $\begin{array}{c}\text { Em desenvolvimento/ } \\
\text { em grande parte } \\
\text { adequada }\end{array}$ & $\begin{array}{c}\text { Bom/ em grande } \\
\text { parte estabelecida }\end{array}$ & $\begin{array}{c}\text { Ótimo/ muito } \\
\text { forte }\end{array}$ \\
\hline
\end{tabular}

Quadro 01 - Distribuição das cores em seus respectivos níveis

Fonte: Zednik (2013)

O quadro de avaliação está estruturado abordando os seguintes elementos: Gestão e Liderança; Planejamento Curricular; Gestão do Processo de Ensino e Aprendizagem; Gestão da Avaliação; Formação Profissional para uso das TIC na Educação; Gestão dos Recursos e Gestão da e-segurança. Os elementos se subdividem em fios condutores, que por sua vez, se subdividem em diferentes aspectos, onde cada aspecto traz a descrição de um quesito traduzido na escala de 1 a 5 . Conforme quadro abaixo.

\begin{tabular}{|c|c|c|}
\hline ELEMENTO & FIO CONDUTOR & ASPECTO \\
\hline \multirow{6}{*}{$\begin{array}{c}\text { Gestão e Liderança } \\
1\end{array}$} & $\begin{array}{l}\text { TIC e a visão da escola } \\
\text { 1a }\end{array}$ & TIC numa visão de médio-longo prazo da Escola (1a-1) \\
\hline & \multirow{3}{*}{$\begin{array}{c}\text { A qualidade da } \\
\text { estratégia para alcançar } \\
\text { visão da escola } \\
\text { 1b }\end{array}$} & A qualidade da estratégia (1b-1) \\
\hline & & Eficácia orçamentária para as TIC (1b-2) \\
\hline & & Sustentabilidade-razoabilidade (1b-3) \\
\hline & \multirow[b]{2}{*}{$\begin{array}{l}\text { Gestão da informação } \\
\text { 1c }\end{array}$} & Gestão estratégica da informação (1c-1) \\
\hline & & $\begin{array}{l}\text { Estratégia de comunicação participação e } \\
\text { compartilhamento (1c-2) }\end{array}$ \\
\hline \multirow{2}{*}{$\begin{array}{l}\text { Gestão do } \\
\text { Currículo } \\
2 \\
\end{array}$} & \multirow{2}{*}{$\begin{array}{l}\text { As TIC presentes no } \\
\text { planejamento de toda } \\
\text { escola } \\
2 \mathrm{a} \\
\end{array}$} & $\begin{array}{l}\text { Planejamento voltado para o desenvolvimento da } \\
\text { capacidade dos alunos de uso criativo das TIC (2a-1) }\end{array}$ \\
\hline & & $\begin{array}{l}\text { Planejamento do uso das TIC para apoiar a } \\
\text { aprendizagem e o ensino com enfoque construtivo (2a-2) }\end{array}$ \\
\hline
\end{tabular}




\begin{tabular}{|c|c|c|}
\hline & & $\begin{array}{l}\text { Planejamento e valorização dos conhecimentos prévios } \\
\text { (2a-3) }\end{array}$ \\
\hline & & $\begin{array}{l}\text { Planejamento das TIC como um meio de promover a } \\
\text { inclusão (2a-4) }\end{array}$ \\
\hline \multirow{7}{*}{$\begin{array}{c}\text { Gestão do processo } \\
\text { Ensino e } \\
\text { Aprendizagem } \\
3\end{array}$} & \multirow{4}{*}{$\begin{array}{c}\text { Processo de ensino e } \\
\text { aprendizagem } \\
\text { 3a }\end{array}$} & $\begin{array}{l}\text { Amplitude do desenvolvimento para a capacidade de uso } \\
\text { das TIC (3a-1) }\end{array}$ \\
\hline & & Qualidade de uso das TIC para ensinar e aprender (3a-2) \\
\hline & & Aprender com as TIC para além da escola (3a-3) \\
\hline & & Avaliação crítica permanente (3a-4) \\
\hline & \multirow{3}{*}{$\begin{array}{l}\text { Experiências de } \\
\text { aprendizagem dos } \\
\text { alunos } \\
\text { 3b }\end{array}$} & $\begin{array}{l}\text { Compreensão dos alunos sobre a sua aprendizagem com } \\
\text { as TIC (3b-1) }\end{array}$ \\
\hline & & Desenvolvimento de habilidades (3b-2) \\
\hline & & Atitudes para a aprendizagem (3b-3) \\
\hline \multirow{3}{*}{$\begin{array}{c}\text { Avaliação do uso } \\
\text { das TIC } \\
4\end{array}$} & \multirow{3}{*}{$\begin{array}{c}\text { Avaliação da } \\
\text { capacidade de uso das } \\
\text { TIC } \\
\text { 4a }\end{array}$} & Autoavaliação e em pares (4a-1) \\
\hline & & $\begin{array}{l}\text { A escola inteira tem metas para melhorar a capacidade } \\
\text { das TIC (4a-2) }\end{array}$ \\
\hline & & $\begin{array}{l}\text { Avaliação do processo de ensino-aprendizagem com as } \\
\text { TIC (6b-3) }\end{array}$ \\
\hline \multirow{3}{*}{$\begin{array}{c}\text { Formação } \\
\text { profissional para } \\
\text { uso das TIC na } \\
\text { educação } \\
5 \\
\end{array}$} & \multirow{3}{*}{$\begin{array}{c}\text { Planejamento para o } \\
\text { desenvolvimento } \\
\text { profissional } \\
5 \mathrm{a}\end{array}$} & $\begin{array}{l}\text { Planejamento profissional e necessidades individuais } \\
(5 a-1)\end{array}$ \\
\hline & & Coaching, mentoring e apoio individual (5a-2) \\
\hline & & $\begin{array}{l}\text { Impacto do desenvolvimento profissional nas atividades } \\
(5 a-4)\end{array}$ \\
\hline \multirow{7}{*}{$\begin{array}{c}\text { Gestão dos } \\
\text { Recursos } \\
\mathbf{6}\end{array}$} & \multirow{7}{*}{$\begin{array}{l}\text { Gestão das TIC } \\
6 \mathrm{a}\end{array}$} & Ambientes físicos $(6 a-1)$ \\
\hline & & $\begin{array}{l}\text { Adequação, suficiência e disponibilidade de hardware } \\
(6 a-2) \text {. }\end{array}$ \\
\hline & & Conectividade interna e online (6a-3) \\
\hline & & Recursos digitais na aprendizagem (6a-4) \\
\hline & & Ambientes de aprendizagem on-line (6a-5) \\
\hline & & Gestão de sistemas de informação (6a-6) \\
\hline & & Suporte técnico (6a-7) \\
\hline \multirow{2}{*}{$\begin{array}{c}\text { Gestão da } e- \\
\text { segurança } \\
7\end{array}$} & \multirow{2}{*}{$\begin{array}{l}\text { Acesso seguro } \\
7^{\mathrm{a}}\end{array}$} & Segurança eletrônica (7a-1) \\
\hline & & Exigências legais (7b-2) \\
\hline
\end{tabular}

Quadro 02 - Estrutura geral do quadro de autoavaliação

Fonte: Zednik (2013)

A Seção 4 corresponde ao footprint (Pegada TE - Tecnologia Educacional), onde busca-se saber os recursos (hardware e software) disponíveis para a escola e quais ela efetivamente utiliza, com a finalidade de comparar com os dados da Seção 3 e contribuir para a análise. Ao final do questionário, o usuário recebe um feedback, gerado pelo sistema, contendo a análise do nível de maturidade e uma representação gráfica como mostra a Figuras 06.

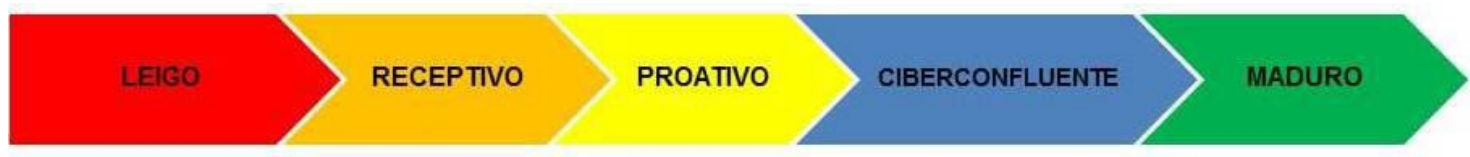

Figura 05 - Nível de maturidade Fonte: Zednik (2013)

Os parâmetros discriminados foram representados em uma régua (Figura 06) de avaliação com uma gradação objetiva (escala de 1 a 5 - onde 1 (um) é o nível mais baixo e 5 (cinco) é o nível mais alto). Esta régua é utilizada para mensurar o nível de maturidade da escola, após média de cada aspecto avaliado.

Os níveis de maturidade representados na Figura 06 demandam uma gradação que indica, quantitativamente, em qual estágio atual a escola avaliada se encontra. Este 
indicador funciona da seguinte maneira: dentro de um critério estabelecido, cada aspecto abordado no questionário recebe do avaliador um nível de maturidade, com base na avaliação da realidade em que se encontra a escola em relação à gestão tecnopedagógica com base nos parâmetros de avaliação descritos no quadro abaixo.

\begin{tabular}{|c|c|c|c|}
\hline \multirow{6}{*}{ ASPECTO } & \multicolumn{2}{|r|}{ NÍVEIS } & PARÂMETRO DE AVALIAÇÃO \\
\hline & 1 & Leigo & $\begin{array}{l}\text { Não existe evidência de ações planejadas ou desconhecem a } \\
\text { questão. }\end{array}$ \\
\hline & 2 & Receptivo & $\begin{array}{l}\text { Há percepção de relevância da questão, mas não há ação } \\
\text { efetiva ou há iniciativas isoladas, não estruturadas ou } \\
\text { coordenadas. Os usuários estão numa posição apenas } \\
\text { receptiva da informação. }\end{array}$ \\
\hline & 3 & Proativo & $\begin{array}{l}\text { Há processo de trabalho formal para as principais atividades } \\
\text { relacionadas à questão. }\end{array}$ \\
\hline & 4 & Ciberconfluente & $\begin{array}{l}\text { Apoia formalmente todas as atividades relacionadas à } \\
\text { questão. }\end{array}$ \\
\hline & 5 & Maduro & É referência na questão. Atende plenamente. \\
\hline
\end{tabular}

O nível 1, LEIGO, evidencia que a escola ainda não desenvolve nenhuma ação dentro do aspecto abordado, ou seja, que a comunidade escolar ainda não percebe a relevância da questão.

O nível 2, RECEPTIVO, demonstra que já existem esboços informais, que algumas pessoas da comunidade escolar já percebem a relevância da questão, mas não existe ainda uma ação planejada, sistematizada e efetiva. Atende de forma desestruturada ou ad hoc a certas demandas relacionadas aos aspectos avaliados. Predominância do uso das TIC para aprendizagem receptiva.

O nível 3, PROATIVO, significa que a escola atende parcialmente e que existe um processo de trabalho formal, direcionado para a realização das principais ações planejadas e estruturadas relacionadas ao aspecto avaliado, que estão sempre se informando, se adaptando, interagindo, extrapolando suas funções, adicionando coisas positivas.

O nível 4, CIBERCONFLUENTE, manifesta claramente que existe registro de todas as atividades relacionadas ao aspecto de forma abrangente e revisado continuamente, ou seja, atende plenamente a todas as demandas relacionadas à questão. Nesse estágio, a escola é capaz de convergir várias culturas e tecnologias com objetivo educacional.

\section{Considerações Finais}

O processo de construção de um questionário não é simples e envolve processos que precisam ser bem estruturados e fundamentados para que não haja prejuízo na análise dos dados obtidos. O embasamento teórico utilizado para a estruturação deste questionário foi focado no E-learning Maturity Model - eMM (Marshall e Mitchell, 2004), nas ferramentas Self Review Framework for ICT, da Naace - Advancing Education Through ICT, no modelo CM360 (Franco et al, 2009), bem como na Teoria da Maturidade-imaturidade, de Chris Argyris (1969); e nas Perspectivas de Transformação de Motta (1999). Tais suportes foram fulcrais na elaboração e construção do modelo e-M - Gestão Tecnopedagógica apresentado neste trabalho.

A delimitação dos objetivos e dos quesitos de um questionário sobre Maturidade da Gestão Tecnopedagógica nos fornece um diferente caminho de pesquisa e facilita a 
proposta de um ideário de desenvolvimento holístico, que seja coerente com a realidade dos respondentes. Os resultados de pesquisas de campo poderão ser enriquecidos com técnicas estatísticas, tal como a análise fatorial, para auxiliar a interpretação $\epsilon$ compreensão dos resultados descritivos obtidos, para identificar linhas de raciocínio $\epsilon$ consequentemente o ideário que permeia as percepções dos respondentes.

Em trabalhos futuros pretendemos descrever o desenvolvimento e funcionamento do sistema dentro de uma visão computacional; o detalhamento proposto por cada elemento; os níveis de maturidade e resultados do pré-teste.

Com o tempo, espera-se que este questionário de autoavaliação cresça, evolua incorpore evidências a partir de avaliações realizadas em diferentes contextos $\epsilon$ instituições e se consolide como rica fonte de pesquisa sobre a gestão da tecnologia nc contexto educacional.

\section{Notas}

i Termo decorrente da "tecnopsicologia", ou "psicotecnologia" de Derrick De Kerckhove.

ii Disponível em: <http://www.utdc.vuw.ac.nz/research/emm/>

iii Disponível em: <http://www.naacesrf.com/>

\section{Referências}

ARGYRIS, C. Personalidade e organização: o conflito entre o sistema e o indivíduo. Rio de Janeiro: Renes, 1968.

COUTINHO, Clara; LISBÔA, Eliana. Sociedade da informação, do conhecimento e da aprendizagem: desafios para educação no século XXI. Revista de Educação, Vol. XVIII, nº 1 , $2011 \mid 5-22$

CUNHA, Luísa Margarida Antunes da. Modelos Rasch e Escalas de Likert e Thurstone na medição de atitudes. Dissertação de Mestrado em Probabilidades e Estatística. Universidade de Lisboa - Faculdade de Ciências, 2007.

CHICKERING, A. W.; GAMSON, Z. F. Seven Principles for Good Practice in Undergraduate Education. AAHE Bulletin, 1987, 39(7), 3-7.

FRANCO, Carlos Eduardo; SANTOS, Marcelino Luis B.; TERRA, José Cáudio C. Gestão de conteúdo $\mathbf{3 6 0}^{\circ}$ : integrando negócios, design e tecnologia. São Paulo: Saraiva, 2009.

IHEP. Quality on line the prepared by: benchmarks for success in internet-based distance education. Washington: The Institute for Higher Education Policy, National Education Association. 2000

MARSHALL, S.; MITCHELL, G. Applying SPICE to e-learning: An e-learning maturity model? Trabalho apresentado na revista Proceedings no VI Australasian Computing Education Conference (ACE2004), Dunedin, New Zealand. Conferences in Research and Practice in Information Technology, Vol. 30. 2004.

Assessing sector e-learning capability with an e-learning maturity model. Edinburgh, UK: Artigo aceito para apresentação e publicação nos anais do ALT-C 2006.

MARTINEZ, Maria Carme; PARAGUAY, Ana Isabel Bruzzi Bezerra. Satisfação e saúde no trabalho: aspectos conceituais e metodológicos. Cadernos de Psicologia Social do Trabalho, 2003, vol. 6, pp. 59-78.

MOTTA, Paulo Roberto. Transformação organizacional: a teoria e a prática de inovar. Rio de janeiro: Qualitymark, 1999.

NAACE. Self-review Framework (SRF). Universidade de Nottingham, 2006. Acesso em: 03 de março de 2012. Disponível em: <http://www.naace.co.uk/ictmark/srf>. 
YANAZE, Leandro Key Higuchi. Tecno-pedagogia: os aspectos lúdicos e pedagógicos da comunicação digital. Dissertação de mestrado. São Paulo: Universidade de São Paulo - Escola de Comunicação e Artes, 2009.

ZEDNIK, Herik; TAROUCO, Liane M. R.; KLERING, Luis R. E-Maturity: entrelaçando gestão, tecnologia e pedagogia. Porto Alegre: Revista Renote - Novas Tecnologias na Educação, V. 10 No 3, dezembro, 2012. 